\title{
Frontal dementia related to thalamic stroke: a case report
}

\author{
Sabrina Realmuto - Valentina Arnao - Antonio Cinturino • \\ Maria Antonietta Mazzola $\cdot$ Simona Talamanca $\cdot$ Marianna Riolo • \\ Ignazio Cusmano $\cdot$ Chiara Cupidi $\cdot$ Tommaso Piccoli
}

Received: 17 February 2012/Accepted: 21 June 2012/Published online: 5 July 2012

(C) Springer-Verlag 2012

Dear Editor,

The behavioral variant of frontotemporal dementia (bvFTD) is characterized by modifications of personality, social behavior and cognition and relies on a neurodegenerative process [1]. However, vascular lesions affecting subcortical structures, mainly the caudate nucleus and thalamus, may result in a clinical syndrome resembling bvFTD $[2,3]$.

We report a case of paramedian thalamic stroke mimicking frontotemporal dementia. A 58-year-old righthanded man was referred to our hospital because of behavioral and cognitive changes formerly diagnosed as bvFTD. His medical history was negative for previous illness and the patient neither smoked nor drank alcohol. In July 2010, the patient suddenly presented with loss of consciousness and was admitted to a nearby hospital. After a few days the decreased level of consciousness resolved and he developed amnesia and personality changes such as disinhibition, apathy and loss of selfactivation. Toxicological exams, routine CSF analysis including total proteins, count cell, glucose and CSF

S. Realmuto $(\square) \cdot$ V. Arnao · A. Cinturino .

M. A. Mazzola - S. Talamanca - M. Riolo - I. Cusmano .

C. Cupidi - T. Piccoli

Dipartimento di Biomedicina Sperimentale e Neuroscienze

Cliniche (BioNeC), Università degli Studi di Palermo,

Via Gaetano La Loggia no 1, Palermo, Italy

e-mail: sabrinarealmuto@gmail.com

C. Cupidi

Centro Regionale di Neurogenetica, ASP Catanzaro,

Via Arturo Perugini, Lamezia Terme, Italy

T. Piccoli

Department of Cognitive Neuroscience, Maastricht University,

Maastricht, The Netherlands culture were normal at admission. CT scan performed within twelve hours the onset of disturbances was also normal. The patient was diagnosed as affected by a conversion disorder. Because of the disturbances, the patient left his job and moved to live with his sister since he was not able to care for himself. After 6 months, the patient was submitted to a neuropsychological screening, showing an impairment of executive functions and amnesia. A diagnosis of frontotemporal dementia was supposed. The patient was referred to our Center in January 2011. The neurological examination was normal. Neuropsychological tests confirmed an alteration of executive functions as well as verbal and visual memory deficits. This was coupled with behavioral disturbances of apathy and depression. Brain MRI showed a mild frontotemporal atrophy with ventricular dilatation but also a left thalamic infarct in the paramedian territory (Fig. 1). The patient started therapy with paroxetine $(20 \mathrm{mg} /$ day $)$ and memantine $(10 \mathrm{mg} /$ day $)$. At 6-month and 1-year, follow-up neuropsychological test showed a further recovery of cognitive functions and behavioral disturbances (Table 1). The caregivers also reported a gradual improvement of disturbances since the onset. Brain CT confirmed thalamic infarction. We made a diagnosis of a thalamic stroke as the cause of dementia, because of the sudden onset and the gradual improvement of the clinical syndrome and the correspondent finding of an ischemic lesion. Routine blood tests, hemocoagulative screening and autoantibody pattern were normal. Electrocardiogram and carotid ultrasonography did not show any alterations. The transthoracic echocardiogram, the transcranial Doppler sonography and the transoesophageal echocardiography detected the patent foramen ovale (PFO) and the patient was started anticoagulant therapy. After 2 years, the 

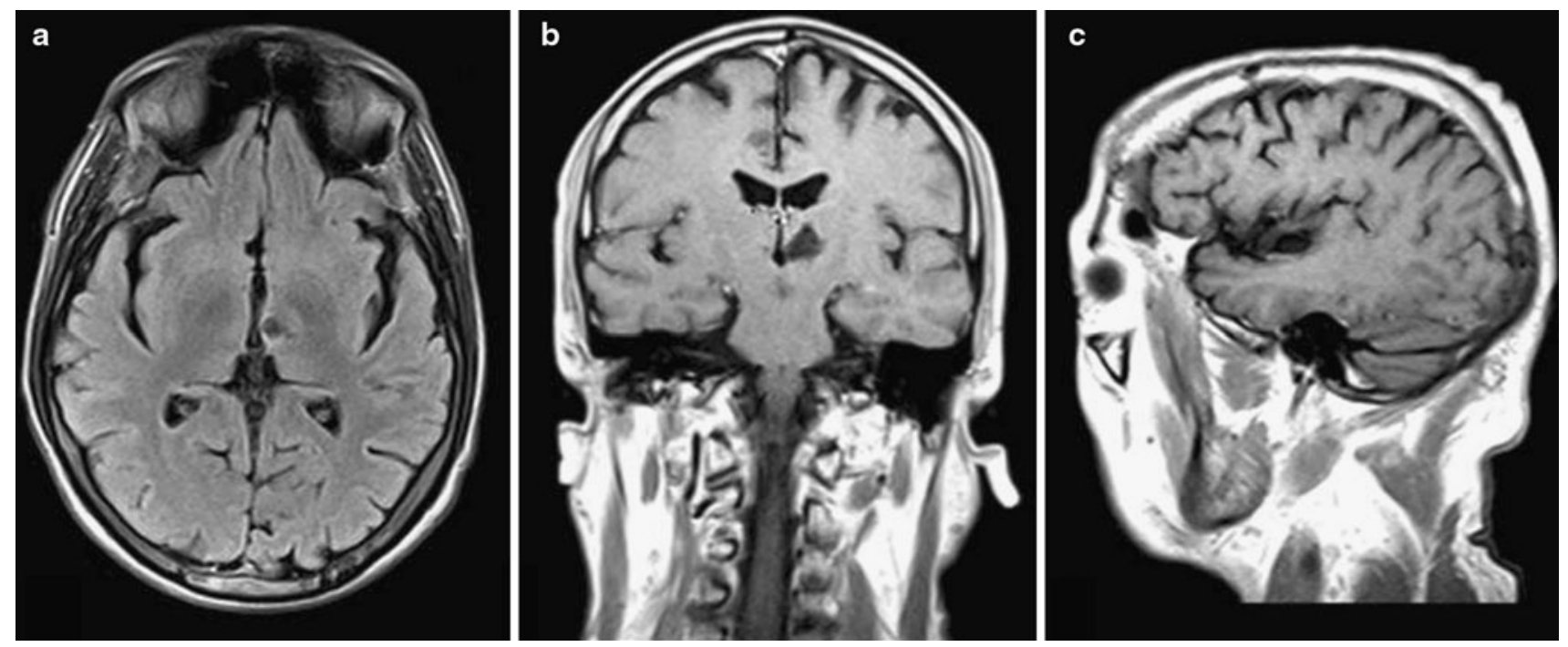

Fig. 1 Brain MRI: axial (a) fluid attenuated inversion recovery (FLAIR) and coronal (b) T1-weighted scans showed the ischemic lesion in the left paramedian thalamic nuclei; sagittal (c) T1-weighted image showed mild frontotemporal atrophy

Table 1 Raw score (adjusted score) of neuropsychological tests at basal examination (T0), at 6 months (T1) and at one-year follow-up (T2)

\begin{tabular}{|c|c|c|c|c|}
\hline Test & T0 & $\mathrm{T} 1$ & $\mathrm{~T} 2$ & Cut-off \\
\hline Mini mental state examination & $22(21.7)$ & $27(26.7)$ & $25(24.7)$ & $\leq 24$ \\
\hline Frontal assessment battery (FAB) & $12(13.41)$ & $15(16.41)$ & $15(16.41)$ & $\leq 12$ \\
\hline Trail making test $\mathrm{A}$ & $110(97)$ & $90(77)$ & $87(74)$ & $>94$ \\
\hline Trail making test $\mathrm{B}$ & $235(187)$ & $220(172)$ & $216(168)$ & $>283$ \\
\hline Visual search & $51(46.5)$ & $44(39.5)$ & $45(40.5)$ & $\leq 31$ \\
\hline Digit span forward & $4(4.5)$ & $4(4.5)$ & $6(6.5)$ & $\leq 3.75$ \\
\hline Corsi span & $4(4.25)$ & $4(4.25)$ & $4(4.25)$ & $\leq 3.25$ \\
\hline Rey auditory verbal learning task immediate recall & $14(14.7)$ & $23(23.7)$ & $18(18.7)$ & $\leq 28.53$ \\
\hline Rey auditory verbal learning task delayed recall & $0(0)$ & $1(1.2)$ & $0(0)$ & $\leq 4.69$ \\
\hline Story recall test & $2.5(4)$ & $2(3.5)$ & $4(5.5)$ & $\leq 4.75$ \\
\hline Letter fluency & $12(19)$ & $8(15)$ & $7(14)$ & $\leq 16$ \\
\hline Category fluency & $22(28)$ & $27(33)$ & $23(29)$ & $\leq 24$ \\
\hline Raven's color progressive matrices & $23(23.8)$ & $31(31.8)$ & $25(25.8)$ & $\leq 18.96$ \\
\hline Rey's complex figure copy & $33(33.5)$ & $29(29.5)$ & $34(34.5)$ & $\leq 28.87$ \\
\hline Rey's complex figure recall & $5(4.25)$ & $10(9.25)$ & $4(3.25)$ & $\leq 9.46$ \\
\hline Constructive apraxia & $13(12.5)$ & $13(12.5)$ & $13(12.5)$ & $\leq 7.75$ \\
\hline Cognitive estimation test (bizarreness score) & $18(6)$ & $15(-)$ & $11(3)$ & $\leq 19(\leq 4)$ \\
\hline Aachener Aphasie test-denomination & $118 / 120$ & $116 / 120$ & $119 / 120$ & - \\
\hline Aachener Aphasie test-oral comprehension & $48 / 60$ & $58 / 60$ & $55 / 60$ & - \\
\hline Neuropsychiatric inventory & 14 & 5 & 6 & - \\
\hline Activity daily living (ADL) & $6 / 6$ & $6 / 6$ & $6 / 6$ & - \\
\hline Instrumental activities of daily living (IADL) & $2 / 5$ & $3 / 5$ & $4 / 5$ & - \\
\hline
\end{tabular}

patient has gradually recovered autonomy in activities of daily living even if he remained apathetic.

Stroke in the paramedian territory of thalamus explains about $35 \%$ of all infarcts in this region and involves mainly the dorsomedian and intralaminar nuclei [4].
Frontal-like syndromes secondary to paramedian strokes are probably due to thalamo-frontal disconnection and behavioral changes consisting of personality abnormalities with disinhibited behavior associated with apathy and amnesia were reported [3]. Amnesia is also a frequent 
sign after paramedian infarcts, but the role of the intralaminar and dorsomedial nuclei is still controversial [5, 6]. Subcortical structures are anatomically and functionally connected with frontal and temporal cortices and damage to those regions often causes frontal lobe dysfunctions. Thalamic vascular lesion should be looked for in differential diagnosis of bvFTD also in patients without apparent vascular risk factors. The reported patient may be diagnosed as cryptogenic stroke (CS), probably caused by PFO. The prevalence of PFO is nearly double in patients with $\mathrm{CS}$ with respect to the general population and the infarct has generally been attributed to a paradoxical embolism [7]. The accurate diagnosis of the vascular origin of frontal dementia in this case has had relevant clinical implications in terms of prognosis and pharmacological treatment.

\section{References}

1. Neary D, Snowden JS, Gustafson L et al (1998) Frontotemporal lobar degeneration: a consensus on clinical diagnostic criteria. Neurology 51:1546-1554

2. Nishio Y, Nakano Y, Matsumoto K et al (2003) Striatal infarcts mimicking frontotemporal dementia: a case report. Eur J Neurol $10: 457-460$

3. Carrera E, Bogousslavsky J (2006) The thalamus and behavior. Effects of anatomically distinct strokes. Neurology 66:1817-1823

4. Von Cramon DY, Hebel N, Schuri A (1985) A contribution to the anatomical basis of thalamic amnesia. Brain 108:993-1008

5. Carlesimo GA, Lombardi MG, Caltagirone C (2011) Vascular thalamic amnesia: a reappraisal. Neuropsychologia 49:777-789

6. Schmahmann JD (2003) Vascular syndromes of the thalamus. Stroke 34:2264-2278

7. Kent MD, Thaler DE (2010) Princeton conference: is PFO a modifiable risk factor for stroke recurrence? Stroke 41(10S):S26S30 